\title{
On Nonlinear Resonant Oscillations of a Rigid Body Generated by Its Conical Precession
}

\section{A. P. Markeev}

The motion of a dynamically symmetric rigid body relative to its center of mass in the central Newtonian gravitational field in a circular orbit is investigated. This problem involves motion (called conical precession) where the dynamical symmetry axis of the body is located all the time in the plane perpendicular to the velocity vector of the center of mass of the body and makes a constant angle with the direction of the radius vector of the center of mass relative to the attracting center. This paper deals with a special case in which this angle is $\pi / 4$ and the ratio between the polar and the equatorial principal central moments of inertia of the body is equal to the number $2 / 3$ or is close to it. In this case, the conical precession is stable with respect to the angles that define the position of the symmetry axis in an orbital coordinate system and with respect to the time derivatives of these angles, and the frequencies of small (linear) oscillations of the symmetry axis are equal or close to each other (that is, the 1:1 resonance takes place).

Using classical perturbation theory and modern numerical and analytical methods of nonlinear dynamics, a solution is presented to the problem of the existence, bifurcations and stability of periodic motions of the symmetry axis of a body which are generated from its relative (in the orbital coordinate system) equilibrium corresponding to conical precession. The problem of the existence of conditionally periodic motions is also considered.

Keywords: resonance, stability, oscillations, canonical transformations

Received July 26, 2018

Accepted August 25, 2018

This work was carried out within the framework of the State Assignment (registration No. AAAA-A17117021310382-5) and was partially supported by the Russian Foundation for Basic Research (project No. 17-01-00123).

Anatoly P. Markeev

anat-markeev@mail.ru

Ishlinsky Institute for Problems in Mechanics RAS

pr. Vernadskogo 101-1, Moscow, 119526 Russia

Moscow Aviation Institute (National Research University)

Volokolamskoe shosse 4, Moscow, 125080 Russia

Moscow Institute of Physics and Technology (State University)

Institutskii per. 9, Dolgoprudnyi, Moscow region, 141700 Russia

RUSSIAN JOURNAL OF NONLINEAR DYNAMICS, 2018, 14(4), 503-518 


\section{Introduction}

A rigid body moving in the central Newtonian gravitational field is considered. The characteristic linear dimensions of the body are assumed to be small in comparison with the distance from the center of mass of the body, $O$, to the attracting center $O_{*}$. This allows the assumption [1] that the rotational motion of the body relative to the center of mass does not influence the motion of the center of mass. We assume that the orbit of the center of mass is circular, and denote the angular velocity of motion of the center of mass in the orbit by $\omega_{0}$.

Let $O X Y Z$ be a trihedron of an orbital coordinate system with the axis $O Z$ directed along the radius vector $\mathbf{O}_{*} \mathbf{O}$ of the center of mass of the body and with the axes $O X$ and $O Y$ directed along the velocity vector of the center of mass and along the normal to the plane of the orbit, respectively. Let us introduce another trihedron, $O x y z$, with axes directed along the principal central axes of inertia of the body, and let $A, B$ and $C$ be the moments of inertia of the body relative to the axes $O x, O y$ and $O z$. The relative orientation of the trihedrons $O X Y Z$ and $O x y z$ will be given by the Euler angles $\psi, \theta, \varphi$ introduced in a standard way.

Assume that the body is dynamically symmetric $(A=B)$. Then the angle of proper rotation, $\varphi$, will be a cyclic coordinate and, as a consequence, the projection $r$ of the absolute angular velocity of the body onto the axis of its dynamical symmetry is constant throughout the motion:

$$
r=\dot{\psi} \cos \theta+\dot{\varphi}-\omega_{0} \cos \psi \sin \theta=r_{0}=\text { const. }
$$

(The dot denotes differentiation with respect to time $t$.)

To describe the rotation of the body relative to its center of mass, we will use the Hamiltonian form of the equations of motion. If we take $\nu=\omega_{0} t$ as an independent variable and nondimensionalize the momenta canonically conjugate to $\psi, \theta$ using the multiplier $A \omega_{0}$, then the Hamiltonian function can be written as [2, 3]:

$$
\begin{aligned}
H & =\frac{1}{2} \frac{p_{\psi}^{2}}{\sin ^{2} \theta}+\frac{1}{2} p_{\theta}^{2}+\left(\alpha \beta \frac{\cos \theta}{\sin ^{2} \theta}+\cos \psi \cot \theta\right) p_{\psi}-\sin \psi p_{\theta}+ \\
& +\frac{1}{2} \alpha^{2} \beta^{2} \cot ^{2} \theta+\alpha \beta \frac{\cos \psi}{\sin \theta}+\frac{3}{2}(\alpha-1) \cos ^{2} \theta, \\
\alpha & =\frac{C}{A}, \quad \beta=\frac{r_{0}}{\omega_{0}} \quad(0<\alpha \leqslant 2, \quad-\infty<\beta<\infty) .
\end{aligned}
$$

The equations given by the Hamiltonian function (1.2) describe the motion of the symmetry axis of the body relative to the orbital coordinate system. If this motion is found, then the rotation of the body about the symmetry axis is defined from (1.1) using a quadrature.

There exist (see, e.g., $[2,3])$ three types of solutions corresponding to the motion of the body in which its symmetry axis is fixed in the orbital coordinate system $\left(\psi=\psi_{0}=\right.$ const, $\theta=\theta_{0}=$ const). For these motions, the symmetry axis: 1) is either always perpendicular to the plane of the orbit, 2) or lies in the plane perpendicular to the radius vector of the center of mass, 3) or lies in the plane perpendicular to the velocity vector of the center of mass of the body. Motions 1), 2) and 3) correspond to regular (respectively, cylindrical, hyperboloidal and conical) precessions of the body in absolute space; the axis of precession is the axis passing through the center of attraction and perpendicular to the plane of the orbit, the angular velocity of precession coincides with the angular velocity $\omega_{0}$ of motion of the center of mass in the orbit, and the angular velocity of proper rotation is defined by the equation $\dot{\varphi}=\left(\beta+\cos \psi_{0} \sin \theta_{0}\right) \omega_{0}$. 
In motions 1), 2) and 3), the symmetry axis of the rigid body stays in absolute space on the surface of a cylinder, a one-sheeted hyperboloid and a cone, respectively.

The problem of stability of the above-mentioned precessions with respect to perturbations of the quantities $\psi, \theta, \dot{\psi}, \dot{\theta}$ has been examined in detail. Relevant references can be found in $[2,3]$.

This paper investigates nonlinear resonant oscillations of the symmetry axis of a body which are generated from its equilibrium point in the orbital coordinate system which corresponds to the conical precession of the body. This precession corresponds to the solution of a system of equations with the Hamiltonian function (1.2) in which

$$
\alpha \beta=(3 \alpha-4) \sin \theta_{0}, \quad \psi_{0}=0, \quad p_{\theta}=p_{\theta_{0}}=0, \quad p_{\psi}=p_{\psi_{0}}=3(\alpha-1) \sin \theta_{0} \cos \theta_{0} .
$$

For this solution, the symmetry axis of the body lies throughout the motion in the plane $O Y Z$ of the orbital coordinate system and makes a constant angle $\theta_{0}$ with the direction of the radius vector of the center of mass of the body.

If the rigid body is dynamically elongated along its symmetry axis $O z$, that is, if the inequality $\alpha<1$ is satisfied, then the Hamiltonian function (1.2) is definite positive in a neighborhood of the conical precession and stability takes place. Inside the stability region $\left\{\alpha<1,0<\theta_{0}<\pi / 2\right\}$ the frequencies $\omega_{1}, \omega_{2}$ of small oscillations of the symmetry axis are defined by the equation

$$
\omega^{4}-\left[7-6 \alpha+9 \alpha(\alpha-1) \sin ^{2} \theta_{0}\right] \omega^{2}+3(\alpha-1)(3 \alpha-4) \cos ^{2} \theta_{0}=0 .
$$

The quantities $\omega_{1}$ and $\omega_{2}$ do not vanish and are different. An exception is one point, $\alpha=2 / 3$, $\theta_{0}=\pi / 4$, at which the frequencies $\omega_{1}$ and $\omega_{2}$ are equal to each other (that is, at this point the 1:1 resonance takes place).

In this paper it is assumed that the quantity $\alpha$ is close or equal to its resonant value $2 / 3$ and the problem of the existence, bifurcations and stability of periodic motions of the symmetry axis is investigated. Some problems concerning conditionally periodic motions are also discussed. The study uses classical perturbation theory, the Deprit - Hori method as well as other numerical and analytical methods and algorithms of nonlinear dynamical systems.

The problem of nonlinear oscillations of Hamiltonian systems in the presence of resonance has a more than 100-year history [4-6]. Relevant references to classical and recent works that contain general theoretical conclusions and their applications to specific problems in mechanics can be found, for example, in [7]. Among the studies on the 1:1 resonance in stable Hamiltonian systems, we mention the papers [8-11] dealing with several model problems important for astrophysics and celestial mechanics. The 1:1 resonance in the problem of motion of a rigid body near its hyperboloidal and cylindrical precessions in a circular orbit has recently been explored in $[7,12]$.

\section{The Hamiltonian function of perturbed motion and its normal form}

Set

$$
\begin{gathered}
\theta=\theta_{0}+\varepsilon \xi_{1}, \quad \psi=\varepsilon \xi_{2}, \quad p_{\theta}=\varepsilon \eta_{1}, \quad p_{\psi}=p_{\psi_{0}}+\varepsilon \eta_{2} \quad(0<\varepsilon \ll 1), \\
\beta=\theta_{0}+\sin \theta_{0}(3 \alpha-4) / \alpha, \quad \theta_{0}=\pi / 4, \quad \alpha=2 / 3+\varepsilon^{2} d .
\end{gathered}
$$


The quantity $\varepsilon^{2} d$ is a deviation of the dimensionless inertial parameter $\alpha$ from its resonant value, which is equal to $2 / 3$. The parameter $\beta$ in (2.1) is such that for any value of $d$ the rigid body can perform a conical precession in which $\theta_{0}=\pi / 4$.

Equations (2.1) define a canonical transformation with the valence $\varepsilon^{-2}$. From (1.2) and (2.1), (2.2) we find that the new canonically conjugate variables $\xi_{i}, \eta_{i}(i=1,2)$ correspond to a Hamiltonian function that can be represented as a power series in the parameter $\varepsilon$ :

$$
\begin{aligned}
H & =\frac{3}{2} \xi_{1}^{2}-2 \xi_{1} \eta_{2}+\frac{3}{4} \xi_{2}^{2}-\xi_{2} \eta_{1}+\frac{1}{2} \eta_{1}^{2}+\eta_{2}^{2}+\varepsilon\left(-\frac{7}{2} \xi_{1}^{3}+5 \xi_{1}^{2} \eta_{2}-\frac{1}{2} \xi_{1} \xi_{2}^{2}-2 \xi_{1} \eta_{2}^{2}+\frac{1}{2} \xi_{2}^{2} \eta_{2}\right)+ \\
& +\varepsilon^{2}\left[\frac{3}{4} d\left(-5 \xi_{1}^{2}+4 \xi_{1} \eta_{2}-\xi_{2}^{2}\right)+\frac{47}{8} \xi_{1}^{4}-\frac{29}{3} \xi_{1}^{3} \eta_{2}+\xi_{1}^{2} \xi_{2}^{2}+4 \xi_{1}^{2} \eta_{2}^{2}-\xi_{1} \xi_{2}^{2} \eta_{2}-\frac{1}{16} \xi_{2}^{4}+\frac{1}{6} \xi_{2}^{3} \eta_{1}\right]+ \\
& +\varepsilon^{3}\left[\frac{9}{4} d\left(3 \xi_{1}^{3}-2 \xi_{1}^{2} \eta_{2}\right)-\frac{77}{8} \xi_{1}^{5}+\frac{193}{12} \xi_{1}^{4} \eta_{2}-\frac{7}{6} \xi_{1}^{3} \xi_{2}^{2}-\frac{20}{3} \xi_{1}^{3} \eta_{2}^{2}+\right. \\
& \left.+\xi_{1}^{2} \xi_{2}^{2} \eta_{2}+\frac{1}{24} \xi_{1} \xi_{2}^{4}-\frac{1}{24} \xi_{2}^{4} \eta_{2}\right]+O\left(\varepsilon^{4}\right) .
\end{aligned}
$$

The canonical univalent change of variables

$$
\xi_{1}=-\frac{\sqrt{6}}{3} u_{1}+\frac{\sqrt{3}}{3} u_{2}, \quad \xi_{2}=\frac{\sqrt{6}}{3} U_{1}+\frac{2 \sqrt{3}}{3} U_{2}, \quad \eta_{1}=\sqrt{3} U_{2}, \quad \eta_{2}=-\frac{\sqrt{6}}{2} u_{1}
$$

reduces the Hamiltonian function (2.3) to the form

$$
G=G^{(0)}+\varepsilon G^{(1)}+\varepsilon^{2} G^{(2)}+\varepsilon^{3} G^{(3)}+O\left(\varepsilon^{4}\right),
$$

where $G^{(0)}$ is a real normal form of the function (2.3) with $\varepsilon=0$ which is the sum of Hamiltonians of two identical harmonic oscillators,

$$
G^{(0)}=\frac{1}{2}\left(u_{1}^{2}+U_{1}^{2}\right)+\frac{1}{2}\left(u_{2}^{2}+U_{2}^{2}\right),
$$

and $G^{(k)}(k=1,2,3)$ in the expansion are functions that are coefficients with $\varepsilon^{k}$ in the expansion (2.3) in which the quantities $\xi_{i}, \eta_{i}(i=1,2)$ have been replaced with their expressions (2.4).

The following transformation $u_{i}, U_{i} \rightarrow q_{i}, p_{i}(i=1,2)$ performs normalization [13] of the Hamiltonian function (2.5) in terms up to the fifth degree in $u_{i}, U_{i}$. This transformation eliminates first- and third-degree terms in $\varepsilon$ and simplifies second-degree terms. The change of variables $u_{i}, U_{i} \rightarrow q_{i}, p_{i}$ was constructed by the Deprit-Hori method [14] modulo third-degree terms in $\varepsilon$. We write out this change of variables, omitting for brevity terms higher than the first degree:

$$
\begin{aligned}
& u_{1}=q_{1}-\varepsilon \frac{\sqrt{6}}{18}\left(2 q_{1}^{2}+2 q_{2}^{2}+3 p_{1}^{2}-2 \sqrt{2} p_{1} p_{2}+2 p_{2}^{2}\right), \\
& u_{2}=q_{2}-\varepsilon \frac{\sqrt{6}}{36}\left(8 q_{1} q_{2}-7 \sqrt{2} q_{2}^{2}-2 \sqrt{2} p_{1}^{2}+8 p_{1} p_{2}-18 \sqrt{2} p_{2}^{2}\right), \\
& U_{1}=p_{1}+\varepsilon \frac{2 \sqrt{6}}{9}\left(q_{1} p_{1}+q_{2} p_{2}\right), \quad U_{2}=p_{2}+\varepsilon \frac{\sqrt{6}}{18}\left(4 q_{1} p_{2}+4 q_{2} p_{1}-7 \sqrt{2} q_{2} p_{2}\right) .
\end{aligned}
$$

In the symplectic polar coordinates $\varphi_{i}, r_{i}$ given by the equations

$$
q_{i}=\sqrt{2 r_{i}} \sin \varphi_{i}, \quad p_{i}=\sqrt{2 r_{i}} \cos \varphi_{i} \quad(i=1,2),
$$


the normalized Hamiltonian function (2.3) can be written as

$$
\begin{aligned}
H & =r_{1}+r_{2}+\varepsilon^{2}\left[-\frac{9}{4} d r_{2}-\frac{1}{6} r_{1}^{2}-\frac{11}{9} r_{1} r_{2}-\frac{13}{18} r_{2}^{2}+\right. \\
& \left.+\frac{\sqrt{2}}{18} r_{1}^{1 / 2} r_{2}^{1 / 2}\left(8 r_{1}+15 r_{2}\right) \cos \left(\varphi_{1}-\varphi_{2}\right)-\frac{1}{9} r_{1} r_{2} \cos 2\left(\varphi_{1}-\varphi_{2}\right)\right]+O\left(\varepsilon^{4}\right) .
\end{aligned}
$$

\section{Simplification of the normal form}

If we neglect the last term in (2.10), we arrive at an approximate system, which, as is easy to check, possesses the integral $r_{1}+r_{2}=$ const. We make use of this fact in order to simplify the normal form (2.10). To do so, we perform a canonical univalent transformation $\varphi_{i}, r_{i} \rightarrow \chi_{i}, R_{i}$ which is given by the equations

$$
\chi_{1}=\varphi_{1}-\varphi_{2}, \quad \chi_{2}=\varphi_{2}, \quad R_{1}=r_{1}, \quad R_{2}=r_{1}+r_{2} .
$$

In the variables $\chi_{i}, R_{i}$ the Hamiltonian function (2.10) can be written as

$$
H=F_{1}+F_{2}+O\left(\varepsilon^{4}\right)
$$

where

$$
\begin{gathered}
F_{1}=-\frac{\varepsilon^{2}}{36}\left[8 R_{1}\left(R_{2}-R_{1}\right) \cos ^{2} \chi_{1}-2 \sqrt{2} R_{1}^{1 / 2}\left(R_{2}-R_{1}\right)^{1 / 2}\left(15 R_{2}-7 R_{1}\right) \cos \chi_{1}-\right. \\
\left.-8 R_{1}^{2}-12 R_{1} R_{2}-81 d R_{1}\right], \\
F_{2}=R_{2}-\frac{\varepsilon^{2}}{36}\left(81 d+26 R_{2}\right) R_{2} .
\end{gathered}
$$

In an approximate system with a Hamiltonian function obtained by neglecting the quantities $O\left(\varepsilon^{4}\right)$ in the function (3.2), the coordinate $\chi_{2}$ is cyclic and there exists the integral $R_{2}=c>0-$ const.

\section{On an auxiliary (model) system with one degree of freedom}

In the above-mentioned approximate system the variables $\chi_{1}, R_{1}$ correspond to a onedegree-of-freedom system with canonical equations of motion given by the function $F_{1}$ from (3.3), in which $R_{2}=c$. If we introduce new variables $\chi, r$ instead of $\chi_{1}, R_{1}$ by the formulae

$$
\chi_{1}=\chi, \quad R_{1}=c r \quad(0 \leqslant r<1)
$$

and take the quantity $\tau=\left(\varepsilon^{2} c / 36\right) \nu$ as an independent variable, then the Hamiltonian function of the model system takes the form

$$
\Gamma=-8 r(1-r) \cos ^{2} \chi+2 \sqrt{2} \sqrt{r(1-r)}(15-7 r) \cos \chi+8 r^{2}+12 r+81 \delta r,
$$

where we have introduced the notation $d=c \delta$.

In the variables $q, p$ defined by the equations

$$
q=\sqrt{2 r} \sin \chi, \quad p=\sqrt{2 r} \cos \chi
$$


we have the following expression for the function (4.2):

$$
\Gamma=\frac{3}{2}(4+27 \delta) q^{2}+\frac{1}{2}(4+81 \delta) p^{2}+2\left(q^{4}+3 q^{2} p^{2}+2 p^{4}\right)+\frac{\sqrt{2}}{2} p\left(30-7 q^{2}-7 p^{2}\right) \sqrt{2-q^{2}-p^{2}} .
$$

The equations of motion of the model system have the first integral

$$
\Gamma=h=\text { const }
$$

On equilibrium points of the model system. The equations of motion of the model system which are given by the Hamiltonian function (4.2) (or (4.4)) admit partial solutions - the equilibrium points $\chi=\chi_{0}, r=r_{0}$ (or $q=q_{0}, p=p_{0}$ ). These solutions are defined by the equations

$$
\frac{\partial \Gamma}{\partial r}=0, \quad \frac{\partial \Gamma}{\partial \chi}=0 \quad\left(\text { or } \quad \frac{\partial \Gamma}{\partial q}=0, \quad \frac{\partial \Gamma}{\partial p}=0\right) .
$$

It follows from (4.6) that the quantity $r$ cannot be zero at equilibrium points and that the second equation of (4.6) can be written as

$$
\left[\frac{\sqrt{2}(15-7 r)}{8 \sqrt{r(1-r)}}-\cos \chi\right] \sin \chi=0 .
$$

The minimal value of the first term in the square brackets is equal to $\sqrt{15}$ (it is attained at $r=15 / 23$ ). Therefore, Eq. (4.7) is equivalent to the equation $\sin \chi=0$ and there exist only two types of equilibrium values $\chi_{0}=\chi_{0}^{(k)}(k=1,2)$ that are physically different from one another:

$$
\chi_{0}^{(1)}=0 \quad \text { and } \quad \chi_{0}^{(2)}=\pi .
$$

Note that the dependence between the equilibrium value $r_{0}$ and the quantity $\delta=\delta_{k}$ is found from the first equation of the system (4.6), in which we need to set $\chi=\chi_{0}^{(k)}(k=1,2)$. Thus, we obtain

$$
\delta_{k}=\delta_{k}\left(r_{0}\right)=-\frac{4}{81}\left(1+8 r_{0}\right)+(-1)^{k} \frac{\sqrt{2}\left(28 r_{0}^{2}-51 r_{0}+15\right)}{81 \sqrt{r_{0}\left(1-r_{0}\right)}} \quad(k=1,2) .
$$

The graphs of functions $\delta_{1}$ and $\delta_{2}$ are shown in Fig. 1. The graph of function $\delta_{1}$ intersects the abscissa axis at the point $r_{0}=0.835$, and the graph of function $\delta_{2}$ intersects it at the point $r_{0}=0.256$. Each of the graphs has two vertical asymptotes: $r_{0}=0$ and $r_{0}=1$.

At the equilibrium point of the $k$ th type the constant $h$ of the integral (4.5) is defined by

$$
h_{k}=h_{k}\left(r_{0}\right)=-16 r_{0}^{2}+(-1)^{k} \frac{\sqrt{2 r_{0}}\left(14 r_{0}^{2}-7 r_{0}-15\right)}{\sqrt{1-r_{0}}} \quad(k=1,2) .
$$

For equilibrium points of both types, $q_{0}=0$ and the quantity $\delta$ is expressed in terms of the equilibrium value $p_{0}$ by the formula

$$
\delta=\delta\left(p_{0}\right)=-\frac{4}{81}\left(1+4 p_{0}^{2}\right)-\frac{\sqrt{2}\left(14 p_{0}^{4}-51 p_{0}^{2}+30\right)}{81 p_{0} \sqrt{2-p_{0}^{2}}} .
$$



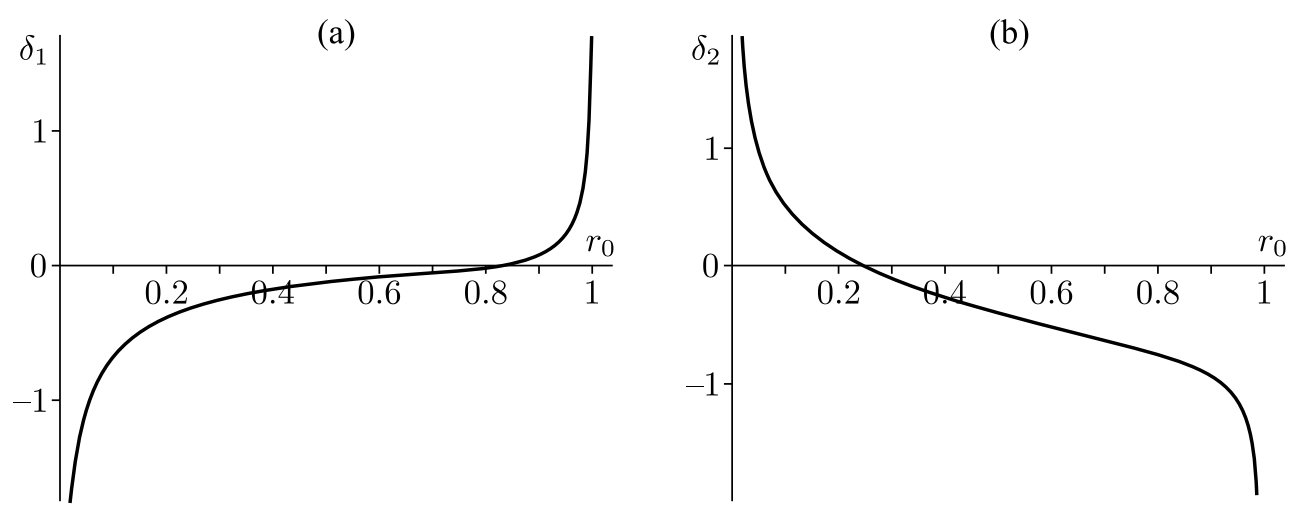

Fig. 1. Dependencies (4.9) between the equilibrium values of $r_{0}$ and the values of $\delta_{1}, \delta_{2}$.

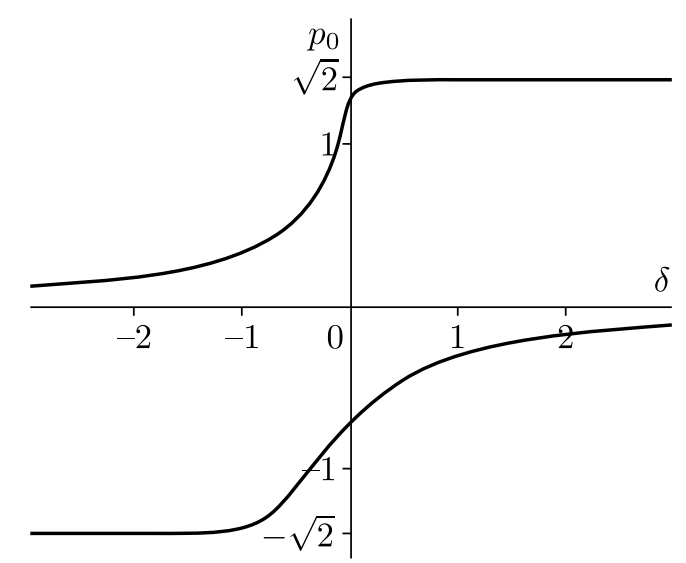

Fig. 2. Equilibrium values of $p_{0}$ as functions of $\delta$.

Equation (4.11) is presented graphically in Fig. 2. The graph intersects the ordinate axis at the points $p_{0}=1.292$ and $p_{0}=-0.716$ and has three horizontal asymptotes: $p_{0}=0, p_{0}=\sqrt{2}$ and $p_{0}=-\sqrt{2}$.

As is seen from Figs. 1 and 2, there exist two equilibrium points for any value of $\delta$ : at the first of them, $\chi_{0}=0, r=r_{0}\left(q_{0}=0, p_{0}=\sqrt{2 r_{0}}\right)$, and at the second, $\chi_{0}=\pi, r=r_{0}\left(q_{0}=0\right.$, $\left.p_{0}=-\sqrt{2 r_{0}}\right)$.

Analysis has shown that for equilibria of both types the roots of the characteristic equation (linearized in a neighborhood of the equilibrium equations of motion of the model system) are purely imaginary. Therefore, the equilibria found in the phase plane correspond to singular points of center type.

\section{On the set of admissible values of the quantities $\delta$ and $h$ in the integral (4.5)}

A straightforward analysis based on the fact that $|\cos \chi| \leqslant 1$ and $0 \leqslant r(1-r) \leqslant 1 / 4$ shows that the set of admissible values of $\delta$ and $h$ consists (see Fig. 3) of two regions $g_{1}$ and $g_{2}$ and their boundaries $\ell$ and $\gamma_{1}, \gamma_{2}$. The boundary curves $\gamma_{k}(k=1,2)$ are given parametrically using the equations $\delta=\delta_{k}\left(r_{0}\right), h=h_{k}\left(r_{0}\right)$, where $0<r_{0}<1$, and functions $\delta_{k}$ and $h_{k}$ are defined 


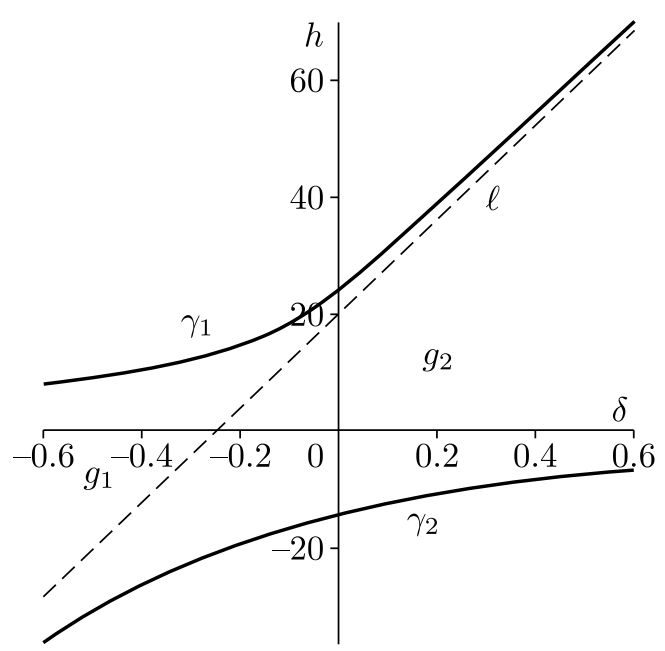

Fig. 3. Regions of admissible values of $\delta$ and $h$ in the integral (4.5).

by Eqs. (4.9) and (4.10). The boundary $\ell$ shown in Fig. 3 is the straight line $h=20+81 \delta$, which separates regions $g_{1}$ and $g_{2}$. It is the common asymptote of the curves $\gamma_{1}$ and $\gamma_{2}$; the abscissa axis $h=0$ is also the common asymptote of these curves. Region $g_{k}$ is a collection of points lying between the asymptote $\ell$ and the curve $\gamma_{k}(k=1,2)$.

For values of $\delta$ and $h$ lying to the left and above the curve $\gamma_{1}$ or to the right and below the curve $\gamma_{2}$, no motion is possible.

The points of the curve $\gamma_{1}$ correspond to equilibria of the first type: $q_{0}=0, p_{0}=\sqrt{2 r_{0}}$, and the points of the curve $\gamma_{2}$ correspond to equilibria of the second type: $q_{0}=0, p_{0}=-\sqrt{2 r_{0}}$.

If the values of $\delta$ and $h$ lie in region $g_{k}(k=1,2)$, then in the phase plane the trajectories of the model system are closed curves which encircle the equilibrium point of the $k$ th type. These two sets of curves are divided by a curve - a separatrix given by the equation

$$
\sqrt{2} p\left(30-7 q^{2}-7 p^{2}\right)-\left[4\left(q^{2}+2 p^{2}\right)+h\right] \sqrt{2-q^{2}-p^{2}}=0 .
$$

The separatrix connects the points $q=-\sqrt{2}, p=0$ and $q=\sqrt{2}, p=0$.

When the parameters $\delta$ and $h$ cross the straight line $\ell$, the phase portrait undergoes a bifurcation. If they cross it, for example, from region $g_{2}$ to region $g_{1}$, then a family of phase curves encircling the singular point $q=0, p=-\sqrt{2 r_{0}}$ disappears and a family of curves encircling the point $q=0, p=\sqrt{2 r_{0}}$ appears.

Figure 4 shows phase portraits for several values of the parameter $\delta$. The phase trajectories are located inside the circles $q^{2}+p^{2}=2$ shown in Fig. 4 by dotted lines. The dashed lines indicate separatrices (5.1).

\section{Families of periodic motions in a complete system}

The equilibrium points $\chi=\chi_{0}, r=r_{0}$ of the model system correspond to the following solutions of the approximate system with the Hamiltonian function $F_{1}+F_{2}$ (see Section 3):

$$
R_{1}=c r_{0}, \quad R_{2}=c, \quad \chi_{1}=\chi_{0}^{(k)}, \quad \chi_{2}=\sigma^{(k)} \quad(k=1,2) .
$$



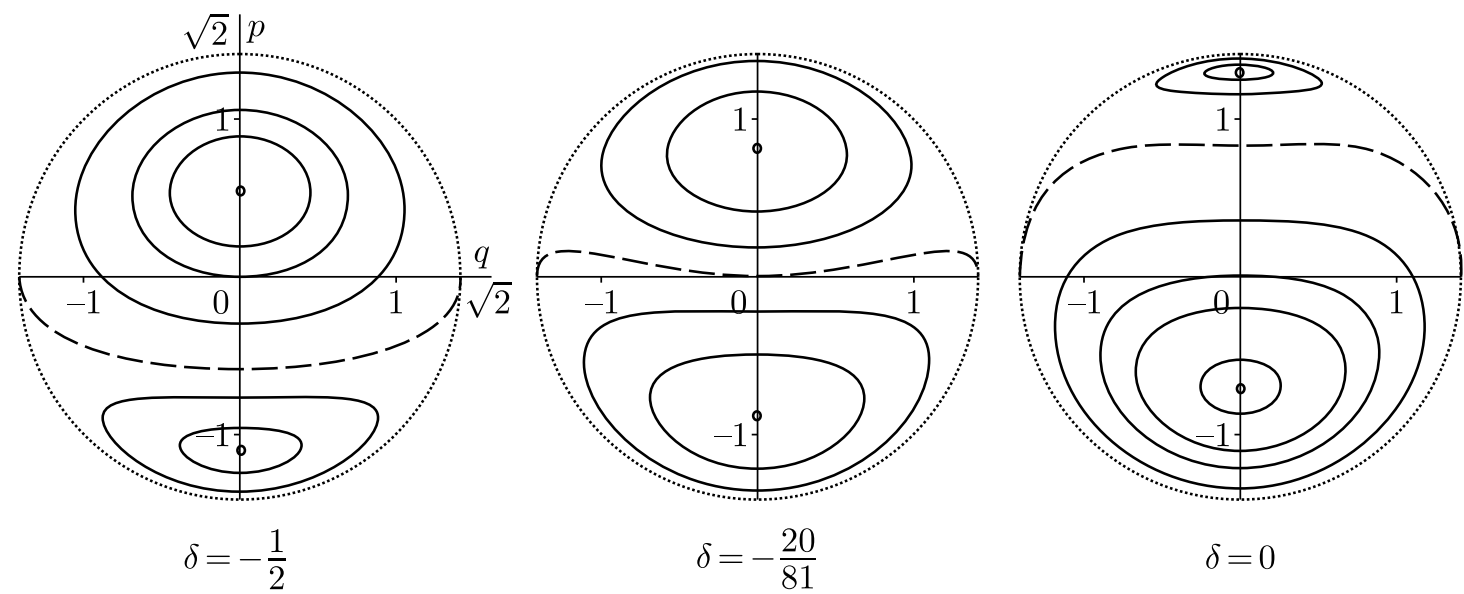

Fig. 4. Phase portraits of the model system.

Here $\chi_{0}^{(k)}$ is given by (4.8), and

$$
\sigma^{(k)}=\omega^{(k)} \nu+\chi_{20} \quad\left(\chi_{20}=\text { const }\right),
$$

where

$$
\omega^{(k)}=1-\frac{\varepsilon^{2} c}{36 \sqrt{r_{0}}}\left[12 \sqrt{r_{0}}\left(4-3 r_{0}\right)+(-1)^{k} 3 \sqrt{2\left(1-r_{0}\right)}\left(5+3 r_{0}\right)\right] .
$$

Using the Poincaré method, as in $[15,16]$, it can be shown that in a complete system with the Hamiltonian function (3.2) there exist two solutions analytic in $\varepsilon$ and tending, as $\varepsilon \rightarrow 0$, to the solutions (6.1). As in the cases of equilibrium of the model system, we shall call them periodic motions of the first type (if in (6.1) $k=1$ ) or of the second type (if $k=2$ ). These families of motions depend on the parameter $c$ and, when $c=0$, degenerate to the equilibrium $\theta_{0}=\pi / 4, \psi_{0}=0$ (see $\left.(2.2)\right)$.

It follows from (6.1) and the change of variables (2.4), (2.6)-(2.9), (3.1) that on the periodic motion of the $k$ th type the quantities $\xi_{1}, \xi_{2}$, which characterize (see (2.2), (2.3)) the deviation of the symmetry axis of the body from its equilibrium point $\theta_{0}=\pi / 4, \psi_{0}=0$ in the orbital coordinate system, are given by the following expressions:

$$
\begin{aligned}
\xi_{1} & =\frac{\sqrt{6 c}}{3}\left[\sqrt{1-r_{0}}+(-1)^{k} \sqrt{2 r_{0}} \sin \sigma_{k}+\right. \\
& +\frac{\varepsilon c}{18}\left[11-7 r_{0}+(-1)^{k} 4 \sqrt{2 r_{0}\left(1-r_{0}\right)}\right]\left(3+\cos 2 \sigma_{k}\right)+O\left(\varepsilon^{2}\right), \\
\xi_{2} & =\frac{2 \sqrt{3 c}}{3}\left[\sqrt{2\left(1-r_{0}\right)}-(-1)^{k} \sqrt{r_{0}}\right] \cos \sigma_{k}- \\
& -\frac{2 \varepsilon c}{9}\left[5-7 r_{0}+(-1)^{k} 4 \sqrt{2 r_{0}\left(1-r_{0}\right)}\right] \sin 2 \sigma_{k}+O\left(\varepsilon^{2}\right) .
\end{aligned}
$$

On trajectories of periodic motions in the plane $\xi_{2}, \xi_{1}$. For a qualitative analysis of the trajectories, we neglect in (6.4) and (6.5) the first- and higher-order terms in $\varepsilon$. Then the approximate expressions for the abscissa $\xi_{2}$ and the ordinate $\xi_{1}$ of the point $M$ of the trajectory of periodic motion of the $k$ th type will be given by the first terms of (6.5) and (6.4).

As we change the quantity $r_{0}$ (or, which is the same, the quantity $\delta$ related to $r_{0}$ by (4.9)), the shape of the trajectory of periodic motion in the plane $\xi_{2}, \xi_{1}$ changes. 
1. We first consider periodic motions of the first type. In the interval $0<r_{0}<1 / 3$ $(\delta<-2 / 9)$ the trajectory is an ellipse with semiaxes $\kappa_{2}$ and $\kappa_{1}$ which are calculated by the formulae

$$
\kappa_{2}=\frac{2 \sqrt{3 c}}{3}\left[\sqrt{2\left(1-r_{0}\right)}+\sqrt{r_{0}}\right], \quad \kappa_{1}=\frac{\sqrt{6 c}}{3}\left|\sqrt{1-r_{0}}-\sqrt{2 r_{0}}\right| .
$$

The ellipse is elongated along the abscissa axis, and point $M$ moves along the ellipse counterclockwise (Fig. 5a). With increasing $r_{0}$ in the above-mentioned interval the semiaxis $\kappa_{2}$ increases from $2 \sqrt{6 c} / 3$ to $2 \sqrt{c}$, and the semiaxis $\kappa_{1}$ decreases from $\sqrt{6 c} / 3$ to zero.

(a)

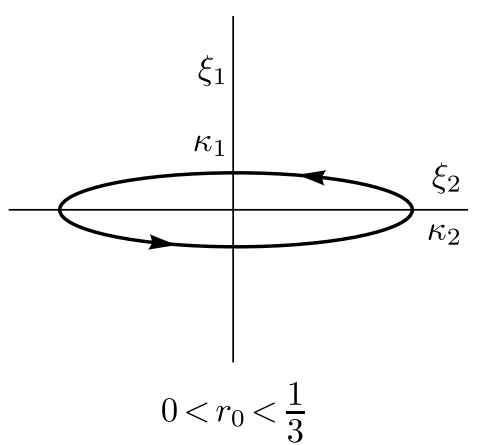

(b)

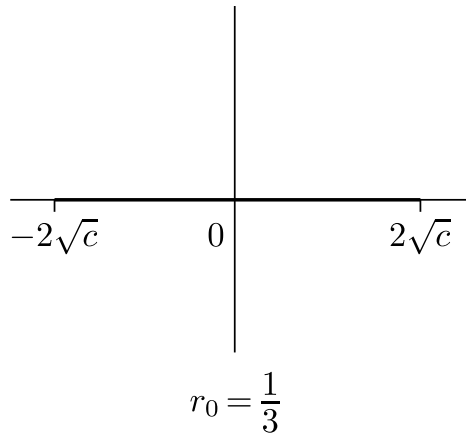

(c)

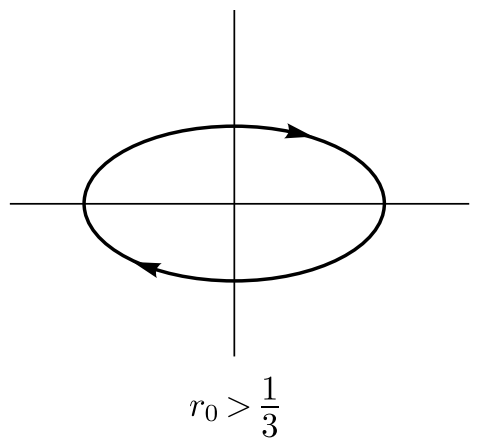

Fig. 5. Periodic motions of the first type in the plane $\xi_{2}, \xi_{1}$.

If $r_{0}=1 / 3(\delta=-2 / 9)$, then the trajectory is a segment of the axis $\xi_{2}$ of length $4 \sqrt{c}$ (Fig. 5b). As $r_{0}$ passes through the value $1 / 3$, the motion of point $M$ along the trajectory reverses direction.

When $r_{0}>1 / 3(\delta>-2 / 9)$, the trajectory is again an ellipse elongated along the axis $\xi_{2}$, point $M$ moves along the ellipse clockwise (Fig. 5 c). With increasing $r_{0}$ the semiaxis $\kappa_{2}$ decreases, and the semiaxis $\kappa_{1}$ increases; in the limit as $r_{0} \rightarrow 1$ the trajectory of point $M$ tends to a circle of radius $2 \sqrt{3 c} / 3$.

2. For motions of the second type the evolution of the shape of trajectories in the plane $\xi_{2}, \xi_{1}$ is somewhat more diverse than in the case of motions of the first type. But as before, the trajectories are, as a rule, ellipses, with the difference that for several values of $r_{0}$ they become circles or degenerate to a straight-line segment. The semiaxes of the ellipses are calculated by the formulae

$$
\kappa_{2}=\frac{2 \sqrt{3 c}}{3}\left|\sqrt{2\left(1-r_{0}\right)}-\sqrt{r_{0}}\right|, \quad \kappa_{1}=\frac{\sqrt{6 c}}{3}\left(\sqrt{1-r_{0}}+\sqrt{2 r_{0}}\right) .
$$

In the interval $0<r_{0}<1 / 9(\delta>4 / 9)$ the ellipse is elongated (as in the case of motions of the first type) along the axis $\xi_{2}$. With increasing $r_{0}$ in this interval $\kappa_{2}$ decreases from $2 \sqrt{6 c} / 3$ to $2 \sqrt{3 c} / 3$, and $\kappa_{1}$ increases from $\sqrt{6 c} / 3$ to $2 \sqrt{3 c} / 3$. Point $M$ moves along the ellipse counterclockwise (Fig. 6a). When $r_{0}=1 / 9(\delta=4 / 9)$, the ellipse becomes a circle of radius $2 \sqrt{3 c} / 3$.

In the interval $1 / 9<r_{0}<2 / 3(-5 / 9<\delta<4 / 9)$ the ellipse is elongated along the axis $\xi_{1}$, and point $M$ moves, as before, counterclockwise (Fig. 6b). With increasing $r_{0}$ the semiaxis $\kappa_{2}$ decreases from $2 \sqrt{3 c} / 3$ to zero, and $\kappa_{1}$ increases from $2 \sqrt{3 c} / 3$ to $\sqrt{2 c}$. When $r_{0}=2 / 3$ $(\delta=-5 / 9)$, the ellipse degenerates to a segment of the axis $\xi_{1}$ of length $2 \sqrt{2 c}$ (Fig. 6 c). 
(a)

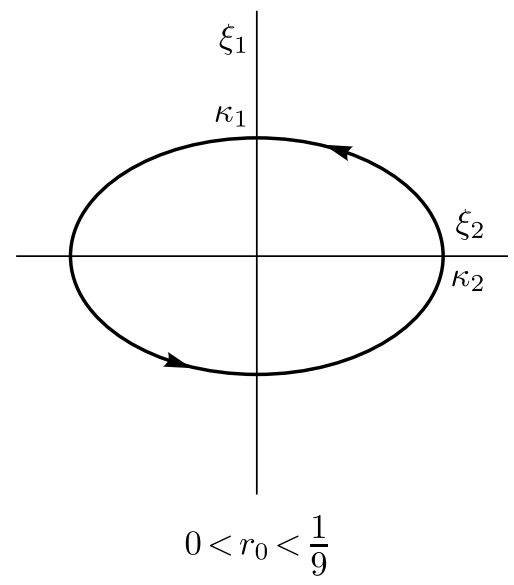

(b)

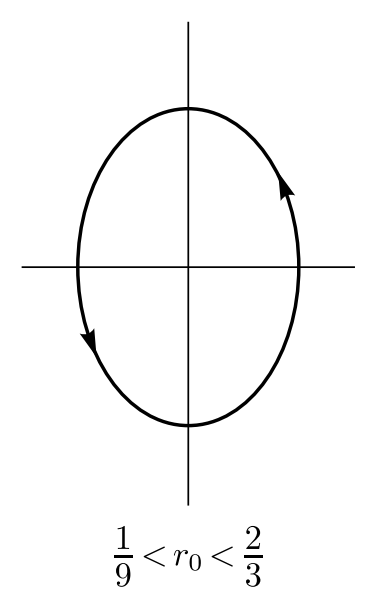

(c)

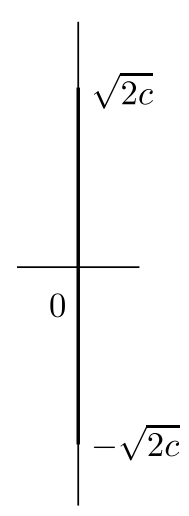

$r_{0}=\frac{2}{3}$ (d)

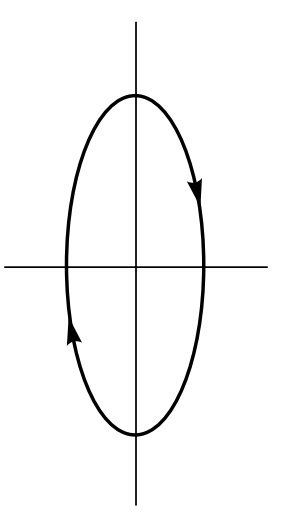

$r_{0}>\frac{2}{3}$

Fig. 6. Periodic motions of the second type in the plane $\xi_{2}, \xi_{1}$.

As $r_{0}$ passes through the value $2 / 3$, the motion of point $M$ along the trajectory reverses direction. When $r_{0}>2 / 3(\delta<-5 / 9)$, the ellipse is elongated along the axis $\xi_{1}$, and point $M$ moves clockwise (Fig. 6d). With increasing $r_{0}$ the semiaxis $\kappa_{2}$ increases and $\kappa_{1}$ decreases and, as a result, as $r_{0} \rightarrow 1$, the trajectory tends to become a circle of radius $2 \sqrt{3 c} / 3$.

On the stability of periodic motions. To analyze the orbital stability of periodic motions, we use the Arnold-Moser theorem [17] on the stability of the Hamiltonian system with two degrees of freedom. A constructive algorithm for such an analysis is set forth in detail in $[18,19]$. We omit the details of calculations and describe only the results obtained therein.

For the stability analysis of periodic motions of the $k$ th type, the quantity $\sigma^{(k)}$ given by $(6.2)$ and (6.3) was taken as an independent variable, and by a suitable choice of real canonically conjugate variables $\varrho_{j}, \vartheta_{j}(j=1,2)$ the Hamiltonian function of perturbed motion was brought to its normal form

$$
\Phi\left(\varrho_{1}, \varrho_{2}, \vartheta_{1}, \vartheta_{2}\right)=\varrho_{1}+\lambda \varrho_{2}+c_{20} \varrho_{1}^{2}+c_{11} \varrho_{1} \varrho_{2}+c_{20} \varrho_{2}^{2}+O\left(\left(\left|\varrho_{1}\right|+\varrho_{2}\right)^{5 / 2}\right) .
$$

In a neighborhood of the trajectory of unperturbed periodic motion the quantity $\varrho_{1}$ can have any sign and $\varrho_{2}$ is nonnegative; on the unperturbed motion $\varrho_{1}=\varrho_{2}=0$. The function (6.8) is analytic in $\varrho_{1},\left|\varrho_{2}\right|^{1 / 2}$ and periodic in $\vartheta_{1}, \vartheta_{2}$.

According to the Arnold-Moser theorem, if the quantity

$$
D=c_{20} \lambda^{2}-c_{11} \lambda+c_{02}
$$

is different from zero, then stability with respect to the variables $\varrho_{1}, \varrho_{2}$ takes place (which in the problem of stability of periodic motion means their orbital stability).

Calculations have shown that the following representation holds for the quantity (6.9) corresponding to periodic motions of the $k$ th type:

$$
D=\varepsilon^{2} \frac{(-1)^{k} 4 P_{1}+\sqrt{2 r_{0}\left(1-r_{0}\right)} P_{2}}{12 Q_{1} Q_{2}}+O\left(\varepsilon^{3}\right) \quad(k=1,2) .
$$


Here the following notation is used:

$$
\begin{aligned}
P_{1} & =1081672704 r_{0}^{13}-8545886208 r_{0}^{12}+29641863168 r_{0}^{11}-58187336256 r_{0}^{10}+ \\
& +67406827536 r_{0}^{9}-38350423944 r_{0}^{8}-8059279372 r_{0}^{7}+32358890456 r_{0}^{6}- \\
& -25287068963 r_{0}^{5}+9140543235 r_{0}^{4}-907382250 r_{0}^{3}-418637250 r_{0}^{2}+ \\
& +137345625 r_{0}-11390625, \\
P_{2} & =3809369088 r_{0}^{13}-34805002752 r_{0}^{12}+139403089152 r_{0}^{11}-324775588608 r_{0}^{10}+ \\
& +493040181744 r_{0}^{9}-521861998968 r_{0}^{8}+405464611788 r_{0}^{7}-234975618790 r_{0}^{6}+ \\
& +90108662031 r_{0}^{5}-8242961049 r_{0}^{4}-12324007230 r_{0}^{3}+5660803800 r_{0}^{2}- \\
& -305704125 r_{0}-193640625, \\
Q_{1} & =\left(5184 r_{0}^{6}-15552 r_{0}^{5}+15552 r_{0}^{4}-3896 r_{0}^{3}-2079 r_{0}^{2}+630 r_{0}+225\right)^{2}, \\
Q_{2} & =81 r_{0}^{2}-242 r_{0}+225 .
\end{aligned}
$$

When $k=1$, the coefficient at $\varepsilon^{2}$ in the expression (6.10) vanishes only in two cases: when $r_{0}=r_{0}^{\prime}=0.557$ and when $r_{0}=r_{0}^{\prime \prime}=0.877$. (The corresponding values are $\delta$ equal to -0.103 and 0.040). Therefore, according to the Arnold-Moser theorem, for values $r_{0}$ that are not equal to $r_{0}^{\prime}$ and $r_{0}^{\prime \prime}$, periodic motions of the first type are orbitally stable if $\varepsilon$ is sufficiently small.

In the case of periodic motions of the second type $(k=2)$, the coefficient at $\varepsilon^{2}$ in (6.10) is zero at $r_{0}=r_{0}^{*}=0.017$ and at $r_{0}=r_{0}^{* *}=0.794(\delta=1.828$ and $\delta=-0.702)$. If $r_{0} \neq r_{0}^{*}$ and $r_{0} \neq r_{0}^{* *}$, then orbital stability takes place at sufficiently small $\varepsilon$.

The problem of stability of periodic motions for values of $r_{0}$ equal to $r_{0}^{\prime}, r_{0}^{\prime \prime}$ (for $k=1$ ) and $r_{0}^{*}, r_{0}^{* *}$ (for $k=2$ ) is left open. Solving this problem requires that the coefficients of terms higher than the fourth degree in $\left|\varrho_{1}\right|^{1 / 2}, \varrho_{2}^{1 / 2}$ be calculated in the expansion (6.8).

\section{On conditionally periodic motions of a complete system}

For values of $\delta$ and $h$ from regions $g_{1}$ and $g_{2}$ (Fig. 3), the motions of an approximate system with the Hamiltonian function $F_{1}+F_{2}$ are oscillations. We examine these oscillations first in an approximate system and then in a complete system with the Hamiltonian function (3.2). We consider only the case of exact resonance, assuming that the dimensionless inertial parameter $\alpha$ in the Hamiltonian function (1.2) is exactly equal to $2 / 3$ and hence the quantity $\delta$ in the functions (4.2) and (4.4) is equal to zero. The phase portrait of the system with the Hamiltonian function (4.4) is shown in the right panel of Fig. 4.

Admissible values of $h$ are given by the inequality $-14.231<h<24.107$. If $h$ lies in the interval $-14.231<h<20$, then the phase trajectories encircle the equilibrium point of the second type $q_{0}=0, p_{0}=-0.716\left(\chi_{0}=\pi, r_{0}=0.256\right)$. If $20<h<24.107$, then the trajectories encircle the equilibrium point of the first type $q_{0}=0, p_{0}=1.292\left(\chi_{0}=0, r_{0}=0.835\right)$. The value $h=20$ corresponds to the separatrix (dashed line in Fig. 4) separating these two sets of closed trajectories.

1. Let $d^{(k)}$ denote the frequency of small linear oscillations of the model system in a neighborhood of the equilibrium of the $k$ th type $(k=1,2)$. Calculations show that $d^{(1)}=20.879$, $d^{(2)}=56.738$.

The frequency $\Omega_{1}^{(k)}$ of nonlinear oscillations of the model system will be a function of $h$. To calculate it, we write one of the equations of the system with the Hamiltonian function (4.2) 
in the form

$$
\frac{d \tau}{d r}=-\left(\frac{\partial \Gamma}{\partial \chi}\right)^{-1}
$$

Substituting the expression $\cos \chi$, obtained from the integral (4.5), in terms of $r$ and $h$,

$$
\cos \chi=\frac{\sqrt{2}(15-7 r-e)}{8 \sqrt{r(1-r)}}, \quad e=\sqrt{81 r^{2}-162 r+225-4 h},
$$

and taking into account the symmetry of phase trajectories relative to the axis $q=0$, we obtain the following expression for period $T$ of nonlinear oscillations:

$$
T=2 \sqrt{2} \int_{r_{\min }}^{r_{\max }} \frac{d r}{e \sqrt{40 r-2 h+(15-7 r) e-e^{2}}} .
$$

The quantities $r_{\min }$ and $r_{\max }$ are the minimal and the maximal values of the variable $r$ in the phase trajectory. For a given $h$ they are the real positive roots of the fourth-degree equation:

$$
648 r^{4}-1944 r^{3}+8(437-4 h) r^{2}-8(225+h) r+h^{2}=0
$$

If we take $\nu$ as an independent variable, then the expression for frequencies $\Omega_{1}^{(k)}$ can be written as

$$
\Omega_{1}^{(k)}=\frac{\varepsilon^{2} c \pi}{18 T}=\frac{\varepsilon^{2} c}{36} d^{(k)} s^{(k)} \quad(k=1,2)
$$

where $s^{(k)}$ is a function of $h$. Their graphs are presented in Fig. 7 .

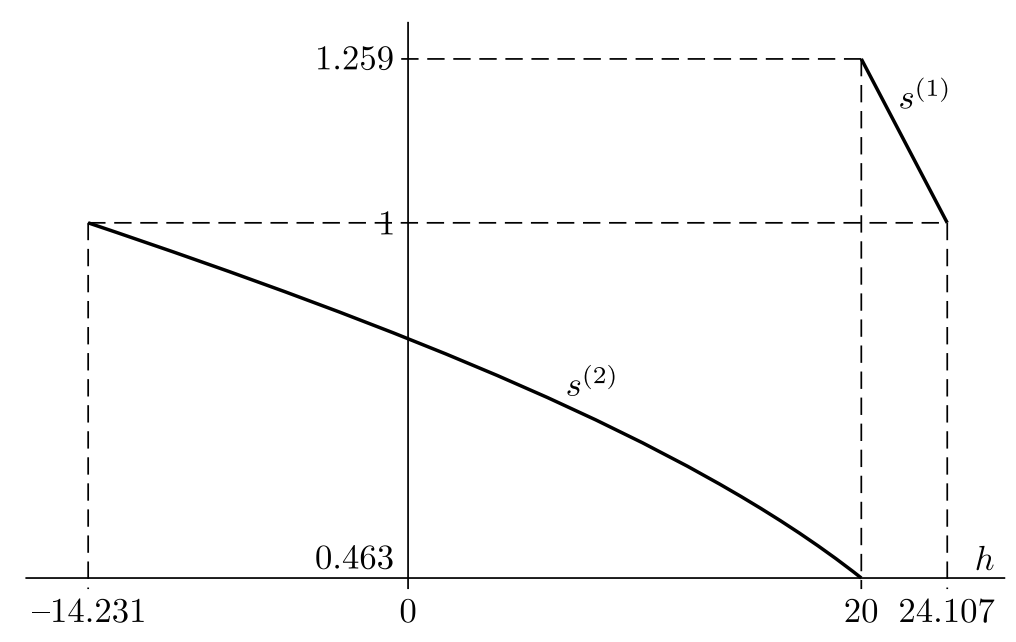

Fig. 7. Graphs of the functions $s^{(k)}(h)(k=1,2)$ from the expressions (7.3) for frequencies of nonlinear oscillations.

2. To investigate nonlinear oscillations in a complete system with the Hamiltonian function (3.2), it is convenient to use canonically conjugate variables $I_{j}, w_{j}(j=1,2)$ which are action-action variables in an approximate system with the Hamiltonian function $F_{1}+F_{2}$. In this system the coordinate $\chi_{2}$ is cyclic, and hence $I_{2}=R_{2}$. The quantity $I_{1}$ is equal to the area, 
divided by $2 \pi$, of a part of the phase plane lying inside a closed phase curve that encloses an equilibrium point of the first or second type:

$$
I_{1}=\frac{1}{2 \pi} \oint R_{1} d \chi_{1}=I_{2} \frac{1}{2 \pi} \oint r d \chi=I_{2} \frac{1}{2 \pi} \oint p(q, h) d q .
$$

It can be seen from these equalities that the quantity $h$ is a function of the ratio $I_{1} / I_{2}$ :

$$
h=\Psi\left(I_{1} / I_{2}\right) .
$$

It follows from (3.3), (3.4), (4.2)-(4.5) that in the variables $I_{j}, w_{j}$ the Hamiltonian function $F_{1}+F_{2}$ of the approximate system does not depend on the angle variables $w_{j}$ and can be written as

$$
H^{*}=H_{0}\left(I_{2}\right)+\varepsilon^{2} H_{2}\left(I_{1}, I_{2}\right)
$$

where

$$
H_{0}\left(I_{2}\right)=I_{2}, \quad H_{2}\left(I_{1}, I_{2}\right)=-\frac{13}{18} I_{2}^{2}+\frac{1}{36} I_{2}^{2} \Psi\left(I_{1} / I_{2}\right) .
$$

The first frequency $\Omega_{1}^{(k)}$ of the approximate system (see (7.3)) can also be calculated by the formula

$$
\Omega_{1}^{(k)}=\frac{\partial H^{*}}{\partial I_{1}}=\frac{\varepsilon^{2}}{36} I_{2} \Psi^{\prime} \quad(k=1,2),
$$

where $\Psi^{\prime}$ is the derivative of the function $\Psi$ with respect to $I_{1} / I_{2}$.

The second frequency $\Omega_{2}^{(k)}$ of oscillations of the approximate system is defined by

$$
\Omega_{2}^{(k)}=\frac{\partial H^{*}}{\partial I_{2}}=1+\frac{\varepsilon^{2}}{36}\left[2 I_{2}(\Psi-26)-I_{1} \Psi^{\prime}\right] \quad(k=1,2) .
$$

If the initial conditions are such that the ratio $\Omega_{1}^{(k)}: \Omega_{2}^{(k)}$ is not a rational number, then the motion in the approximate system will be conditionally periodic (of the $k$ th type) with frequencies $\Omega_{1}^{(k)}$ and $\Omega_{2}^{(k)}(k=1,2)$.

3. The Hamiltonian function (3.2) of a complete system in the variables $I_{j}, w_{j}$ is given by

$$
H=H_{0}\left(I_{2}\right)+\varepsilon^{2} H_{2}\left(I_{1}, I_{2}\right)+\varepsilon^{4} H_{4}\left(I_{1}, I_{2}, w_{1}, w_{2} ; \varepsilon\right) .
$$

It is analytic in all its variables and $2 \pi$-periodic in $w_{1}, w_{2}$. In the limit as $\varepsilon=0$, the Hamiltonian function (7.9) depends only on the action variable $I_{2}$. Therefore, in the system realize the case of proper degeneracy [17].

The following inequalities hold for the function (7.9):

$$
\frac{\partial H_{0}}{\partial I_{2}} \neq 0, \quad \frac{\partial H_{2}}{\partial I_{1}} \neq 0, \quad \frac{\partial^{2} H_{2}}{\partial I_{1}^{2}} \neq 0
$$

The first two inequalities follow immediately from (7.6), (7.7) and (7.3) (see also Fig. 7). Let us check how the third inequality is satisfied.

From (7.7) and (7.3), (7.4) we have the following chain of equations:

$$
\frac{\partial^{2} H_{2}}{\partial I_{1}^{2}}=\frac{\varepsilon^{2} I_{2}}{36} \frac{d \Psi^{\prime}}{d I_{1}}=\frac{\varepsilon^{2} I_{2}}{36} d^{(k)} \frac{d s^{(k)}}{d I_{1}}=\frac{\varepsilon^{2} I_{2}}{36} d^{(k)} \frac{d s^{(k)}}{d h} \frac{d h}{d I_{1}}=\frac{\varepsilon^{2} d^{(k)^{2}}}{36} s^{(k)} \frac{d s^{(k)}}{d h} .
$$




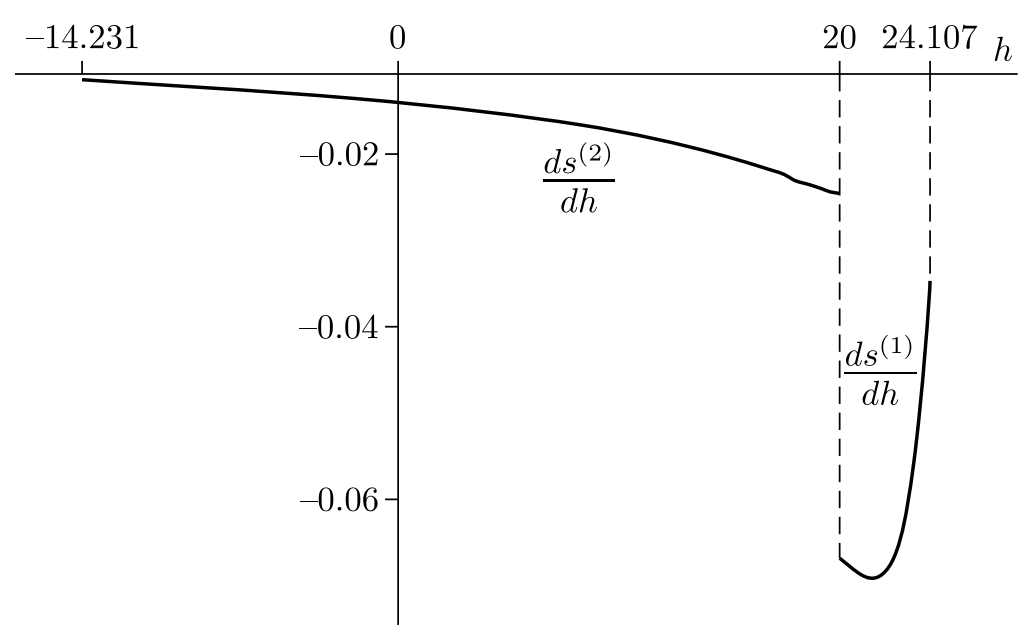

Fig. 8. Graphs of the derivatives $d s^{(k)} / d h(k=1,2)$.

Since the quantities $d^{(k)}$ and $s^{(k)}$ do not vanish, the third inequality of (7.10) is equivalent to the difference from the zero of the derivatives $d s^{(k)} / d h(k=1,2)$. These derivatives were found numerically using the equation $s^{(k)}=2 \pi /\left(d^{(k)} T\right)$ following from (7.3), where $T$ is the integral (7.2) depending on the parameter $h$. It turned out that both derivatives are different from zero (are zero). Their graphs are shown in Fig. 8.

Since all three inequalities (7.10) are satisfied, the motion of the complete system $[17,20]$ with the Hamiltonian function (3.2) is conditionally periodic with frequencies $\Omega_{1}^{(k)}$ and $\Omega_{2}^{(k)}$ $(k=1,2)$. Only a portion of order $\exp \left(-a_{1} \varepsilon^{2}\right)$, where $a_{1}=$ const $>0$, of the phase space is not filled with conditionally periodic trajectories. The quantities $I_{j}(j=1,2)$ are close to their initial values for all $\nu$ :

$$
\left|I_{j}(\nu)-I_{j}(0)\right|<a_{2} \varepsilon^{2} \quad\left(a_{2}-\text { const }\right) .
$$

\section{References}

[1] Beletskii, V. V., Motion of an Artificial Satellite about Its Center of Mass, Jerusalem: Israel Program for Scientific Translations, 1966.

[2] Beletskii, V.V., Satellite's Motion about Center of Mass in a Gravitational Field, Moscow: MGU, 1975 (Russian).

[3] Markeev, A. P., Linear Hamiltonian Systems and Some Problems of Stability of the Satellite Center of Mass, Izhevsk: R\&C Dynamics, Institute of Computer Science, 2009 (Russian).

[4] Korteweg, D. J., Sur certaines vibrations d'ordre supérieur et d'intensité anormale — vibrations de relations, - dans les mécanismes à plusieurs degrés de liberté, Arch. Néerl. sci. exactes et natur. Sér. 2, 1898, vol. 1, pp. 229-260.

[5] Beth, H. I. E., Les oscillations autour d'une position dans le cas d'existence d'une relation linéaire simple entre les nombres vibratoires, Arch. Néerl. sci. exactes et natur. Sér. 2, 1910, vol. 15, pp. $246-283$.

[6] Beth, H. I.E., Les oscillations autour d'une position dans le cas d'existence d'une relation linéaire simple entre les nombres vibratoires (suite), Arch. Néerl. sci. exactes et natur. Sér. 3A, 1912, vol.1, pp. 185-213.

[7] Markeyev, A.P., Non-Linear Oscillations of a 1 : 1 Resonance Hamiltonian System, J. Appl. Math. Mech., 2011, vol. 75, no.6, pp.631-646; see also: Prikl. Mat. Mekh., 2011, vol. 75, no. 6, pp.901-922. 
[8] Hénon, M. and Heiles, C., The Applicability of the Third Integral of Motion: Some Numerical Experiments, Astronom. J., 1964, vol.69, no. 1, pp. 73-79.

[9] Roels, J., An Extension to Resonant Cases of Liapunov's Theorem Concerning the Periodic Solutions near a Hamiltonian Equilibrium, J. Differential Equations, 1971, vol. 9, no. 2, pp. 300-324.

[10] Braun, M., On the Applicability of the Third Integral of Motion, J. Differential Equations, 1973, vol. 13, no. 2, pp. 300-318.

[11] Breiter, S. and Elipe, A., Pseudo-Oscillator with a Quartic Perturbation, Mech. Res. Comm., 2001, vol. 28, no. 2, pp. 119-126.

[12] Kholostova, O. V. and Safonov, A. I., Investigation of the Motions of an Autonomous Hamiltonian System at a 1 : 1 Resonance, Regul. Chaotic Dyn., 2017, vol. 22, no. 7, pp. 792-807.

[13] Birkhoff, G. D., Dynamical Systems, Providence, R.I.: AMS, 1966.

[14] Giacaglia, G.E.O., Perturbation Methods in Non-Linear Systems, Appl. Math. Sci., vol. 8, New York: Springer, 1972.

[15] Markeyev, A. P., The Critical Case of a Pair of Zero Roots in a Two-Degree-of-Freedom Hamiltonian System, J. Appl. Math. Mech., 1998, vol.62, no.3, pp.341-349; see also: Prikl. Mat. Mekh., 1998, vol. 62 , no. 3, pp. 372-382.

[16] Markeev, A.P., On Stability and Non-Linear Oscillations of Hamiltonian System in a Resonant Case, Mech. Solids, 1998, vol.33, no.4, pp. 32-41; see also: Izv. Ross. Akad. Nauk. Mekh. Tverd. Tela, 1998, no. 4, pp. 38-49.

[17] Arnol'd, V.I., Kozlov, V.V., and Neřshtadt, A.I., Mathematical Aspects of Classical and Celestial Mechanics, 3rd ed., Encyclopaedia Math. Sci., vol. 3, Berlin: Springer, 2006.

[18] Markeev, A. P., Nonlinear Oscillations of Sympathetic Pendulums, Nelin. Dinam., 2010, vol. 6, no. 3, pp. 605-621 (Russian).

[19] Markeyev, A.P., An Algorithm for Normalizing Hamiltonian Systems in the Problem of the Orbital Stability of Periodic Motions, J. Appl. Math. Mech., 2002, vol.66, no.6, pp. 889-896; see also: Prikl. Mat. Mekh., 2002, vol.66, no.6, pp. 929-938.

[20] Neishtadt, A. I., Estimates in the Kolmogorov Theorem on Conservation of Conditionally Periodic Motions, J. Appl. Math. Mech., 1981, vol.45, no.6, pp.766-772; see also: Prikl. Mat. Mekh., 1981, vol. 45, no. 6 , pp. 1016-1025. 